\title{
Frequency and predictors of colonization of the respiratory tract by VIM-2-producing Pseudomonas aeruginosa in patients of a newly established intensive care unit
}

\begin{abstract}
Correspondence Matthew E. Falagas m.falagas@aibs.gr
\end{abstract}

Received 8 May 2006 Accepted 6 July 2006

\author{
Maria Horianopoulou, ${ }^{1,2}$ Nicholas J. Legakis, ${ }^{3}$ Maria Kanellopoulou, ${ }^{4}$ \\ Sotiris Lambropoulos, ${ }^{1}$ Athanassios Tsakris ${ }^{3}$ and Matthew E. Falagas ${ }^{5,6}$ \\ ${ }^{1,2,4}$ Intensive Care Unit ${ }^{1}$, Department of Medicine ${ }^{2}$ and Laboratory of Clinical Microbiology ${ }^{4}$, \\ Sismanoglio General Hospital, Athens, Greece \\ ${ }^{3}$ Department of Microbiology, Medical School, University of Athens, Athens, Greece \\ ${ }^{5}$ Alfa Institute of Biomedical Sciences (AIBS), 9 Neapoleos Street, Marousi 151 23, Athens, \\ Greece \\ ${ }^{6}$ Department of Medicine, Tufts University School of Medicine, Boston, MA, USA
}

The aim of this study was to examine the frequency and predictors of colonization of the respiratory tract by metallo- $\beta$-lactamase (MBL)-producing Gram-negative bacteria in patients admitted to a newly established intensive care unit (ICU) of a tertiary care hospital. Specimens of tracheobronchial aspirates for microbiological studies were obtained every day for the first 3 days of the ICU stay and subsequently every third day for the rest of the ICU stay. PCR analysis and nucleotide sequencing were performed to identify bacteria that had MBL genes. Thirty-five patients (20 male, 15 female) were hospitalized during the initial 3 month period of functioning of the ICU. Colonization of the lower respiratory tract by Gram-negative bacteria was found in 29 of 35 patients ( $83 \%$ ) during the first 6-20 days (median 13 days) following admission to the ICU (13 patients with Acinetobacter baumannii, ten with Pseudomonas aeruginosa, three with Enterobacter aerogenes, two with Klebsiella pneumoniae and one with Stenotrophomonas maltophilia). Six of 29 patients $(21 \%)$ colonized with Gram-negative bacteria had blaviM-2-positive $P$. aeruginosa isolates; one of these patients developed clinical infection due to this micro-organism. Previous use of carbapenems $(P=0.01)$ or other $\beta$-lactams $(P=0.03)$, as well as a stay in the ICU of $>20$ days $(P<0.001)$, were associated with colonization with blaviM-2-producing $P$. aeruginosa. In conclusion, colonization by Gram-negative bacteria of the respiratory tract of patients in this newly established ICU was common ( $83 \%$ ). Use of $\beta$-lactams, including carbapenems, was associated with subsequent colonization of the respiratory tract with MBL-positive $P$. aeruginosa.

\section{INTRODUCTION}

Colonization of the skin and mucosal surfaces of the human body by various micro-organisms is a natural process commencing at birth. The micro-organisms that colonize the epithelial and mucosal cells comprise the normal flora, which is made up of resident and transient flora (Bonten \& Weinstein, 1996; Itokazu et al., 1996; Jarvis, 1996). The resident flora is complex and consists of a plethora of organisms, with a predominance of bacteria and fungi. The exact micro-organisms vary from patient to patient, but

Abbreviations: CLSI, Clinical and Laboratory Standards Institute; ICU, intensive care unit; MBL, metallo- $\beta$-lactamase; SAPS, simplified acute physiology score. remain more or less constant in the undisturbed, nonmanipulated host.

The transient microbial flora refers to the species of microorganisms that colonize various parts of the human body for short periods of time. Colonization with transient flora usually lasts from a few hours up to several weeks. These micro-organisms originate from the environment and remain in balance with those of the resident flora for some time. The transient flora may lead to infection, especially if there are changes in the resident flora. The natural balance of micro-organisms on the human skin and mucosal surfaces is disturbed when a patient is admitted to hospital, especially in the environment of the intensive care unit (ICU). Patients are colonized by hospital bacteria, which may lead to serious infection (Flaherty \& Weinstein, 
1996). Previous studies have shown that colonization of the respiratory and urinary tracts usually occurs within a few days in the majority of patients, if not all, in the ICU (Johanson et al., 1972; Kerver et al., 1988; Trautmann et al., 2001). Several factors have been associated with the development of colonization of the mucosal surfaces of patients at an early stage of their ICU stay; the presence of bacteria in the ICU environment is considered to be among them.

During the last decade, carbapenem resistance among Gram-negative bacteria has been attributed partly to the production of acquired metallo- $\beta$-lactamases (MBLs), among which the most prevalent are IMP- and VIM-type enzymes, whilst SPM, GIM and SIM types have been scarcely reported from some regions. All of these enzymes except for SPM are encoded on class 1 integrons (some IMP genes are also encoded on class 3 integrons). The $b l a_{\mathrm{VIM}}$ gene was first detected in 1996 in Portugal and then in Italy in 1997, whilst the first report was in 1999 (Lauretti et al., 1999). A variant gene $\left(b l a_{\mathrm{VIM}-2}\right)$ with $90 \%$ nucleotide identity with $b l a_{\mathrm{VIM}-1}$ was detected some months later in France (Poirel et al., 2000). Several variants of the $b a_{\mathrm{VIM}}$ genes have since been detected. For example, $b l_{\mathrm{VIM}-3}$ has two nucleotide differences with $b l a_{\mathrm{VIM}-2}$, whilst $b l a_{\mathrm{VIM}-4}$ has one nucleotide difference with VIM-1, resulting in an amino acid change of serine to arginine at position 175 (Andrade et al., 2003; Pournaras et al., 2002, 2003; Walsh et al., 2005; Yan et al., 2001). To date, more than 12 VIM variants have been identified, ranging in identity from 77 to $99 \%$. These consist of three separate groups, the VIM-1 group, the VIM-2 group and VIM-7 (consisting of one member); VIM-7 is the most divergent, with many substitutions of amino acids. Acquired MBLs readily hydrolyse most $\beta$-lactams, including carbapenems, and have been detected mostly among carbapenem-resistant nosocomial Pseudomonas aeruginosa strains. In our region, several studies have shown a wide dissemination of VIM-type MBLs among carbapenemresistant Gram-negative bacteria, whilst other MBLs have not been detected (Pournaras et al., 2002, 2003; Walsh et al., 2005).

We had the chance to work in a hospital (Sismanoglio General Hospital, Athens, Greece) when a new ICU was being established. Thus, we aimed to study the colonization of the respiratory tract in patients admitted to this newly established ICU during its first period of function, focusing on Gram-negative bacteria, to evaluate how quickly colonization occurred and by which bacteria, as well to evaluate the frequency and predictors of colonization with MBL-producing Gram-negative bacteria.

\section{METHODS}

Patient population. We studied all patients who were admitted to a newly established ICU of a tertiary care hospital over the first 3 month period of its operation. Patients who were colonized with VIM-2-producing $P$. aeruginosa had not been previously admitted to a facility that harboured VIM-2-producing isolates. The ICU had eight patient beds; four were in isolated rooms (with one patient bed per room). Demographic and clinical data, including information regarding the new simplified acute physiology score (SAPS II) of the studied patients, were collected on their admission to the ICU (Le Gall et al., 1993). The study protocol was reviewed in detail regarding ethical issues and the expected scientific value of the research findings, and was subsequently approved by the staff committee of the Department of Critical Care overseeing clinical studies.

Definitions of colonization and infection. Diagnosis of pneumonia required at least one of the following: new or progressive and persistent infiltrate, consolidation, cavitation or pleural effusion, fever $>38^{\circ} \mathrm{C}$ with no other recognized cause or abnormal white blood cell count [leukopenia $\left(<4000\right.$ white blood cells $\mathrm{mm}^{-3}$ ) or leukocytosis $\left(\geqslant 12000\right.$ white blood cells $\left.\mathrm{mm}^{-3}\right)$ ]; and at least two of the following: new onset of purulent sputum or change in character of sputum, increased respiratory secretions or increased suctioning requirements, new onset or worsening of cough or dyspnoea or tachypnoea, rales or bronchial breath sounds or worsening gas exchange. Isolation of micro-organisms from the tracheobronchial tree without the fulfilment of any of the combinations of the above criteria was attributed to colonization of the respiratory tract.

Clinical specimen cultures. Tracheobronchial aspirate specimen cultures were obtained every day for the first 3 days following admission and subsequently every third day. An oral consent for taking the above-mentioned specimens was given by the patients or their relatives (when patients were unable to communicate) to one of the authors of the study (M.H.).

Microbiological studies. Identification of the micro-organisms to the species level was performed with the PASCO automated system (Difco Laboratories) according to the manufacturer's instructions, and by testing for various properties using key tests prepared in house. We focused only on Gram-negative bacteria, based on the design of our study. The Gram-negative isolate with the highest number of c.f.u. $\mathrm{ml}^{-1}$ of the tested specimen was considered to be the main Gram-negative colonizing micro-organism and was examined further with antimicrobial-susceptibility testing. The MICs of anti-pseudomonal drugs (ceftazidime, piperacillin/tazobactam), except carbapenems, were determined with the PASCO microdilution system applying the criteria suggested by the Clinical and Laboratory Standards Institute (CLSI). No in vitro susceptibility testing of the studied isolates to ertapenem or other carbapenems under development was performed. Susceptibility to imipenem and meropenem was determined by agar disc diffusion in accordance with the CLSI recommendations. Isolates that were found to be resistant to carbapenems by this technique were tested further using MuellerHinton agar containing serial twofold dilutions of antibiotic and a final inoculum of $10^{4}$ c.f.u. per spot to determine the MICs of imipenem, meropenem and other anti-pseudomonal drugs, based on a CLSI-recommended agar dilution method.

Molecular assays. A $b l a_{\mathrm{IMP}}$-specific product $(587 \mathrm{bp}$ ) was amplified by PCR from crude DNA extracts, obtained after heating bacterial suspensions in a $100^{\circ} \mathrm{C}$ heat block for $15 \mathrm{~min}$, using published primers and conditions (Itokazu et al., 1996), whilst detection of $b l a_{\mathrm{VIM}}$ alleles was performed with two different pairs of consensus primer sequences that amplify internal fragments of 261 and $510 \mathrm{bp}$, respectively (Poirel et al., 2000; Tsakris et al., 2000). PCR was also performed on the $b a_{\mathrm{VIM}}$-positive isolates using specific oligonucleotide primers designed to amplify the entire sequences of the $b l_{\mathrm{VIM}-1}$ (920 bp product) and $b l_{\mathrm{VIM}-2}$ (865 bp product) genes (Senda et al., 1996). The amplicons were purified using a Qiaex II gel extraction kit (Qiagen) and used as templates for sequencing of both strands using an ABI Prism 377 DNA sequencer (Perkin-Elmer). In PCR assays, $P$. aeruginosa 174 strain (Mavroidi et al., 2000) and $P$. aeruginosa 101/1477 strain (kindly provided by $\mathrm{Dr}$ N. Woodford, 
Antibiotic Monitoring and Reference Laboratory, Central Public Health Laboratory, London, UK) were used as positive controls for detecting $b l a_{\mathrm{VIM}}$ and $b l a_{\mathrm{IMP}}$, respectively. As an internal control, primers for the cephalosporinase gene (de Champs et al., 2002), which is constantly present in Pseudomonas, were used.

Statistical analysis. Statistic analyses were performed using SPSS software version 11 (SPSS). Discrete data were expressed as percentages and compared using a $\chi^{2}$ test. A comparison of the distribution of quantitative variables was performed with the $t$ test and the Wilcoxon rank sum test (for normally and normally distributed variables, respectively). $P$ values were based on two-tailed test results.

\section{RESULTS}

Thirty-five patients (20 male, 15 female) were admitted to the ICU during the first 3 months of its operation. The demographic and clinical characteristics of the patients are shown in Table 1. Thirty-three of the 35 studied patients were intubated and mechanically ventilated for at least a part of their ICU stay. None of the newly admitted patients had positive cultures on admission to the ICU. All patients had a urinary catheter.

Bacteria were grown from 120 cultures of 420 specimens of tracheobronchial aspirates. In four of these 120 positive cultures, the isolates were considered to be pathogens, i.e. the aetiological agents of clinical infection rather than colonizing agents, based on the criteria described above.

Table 1. Demographic and clinical features of the 35 ICU patients studied

Results are given as number of patients (\%), unless indicated otherwise.

\begin{tabular}{|lc|}
\hline Characteristic & Result \\
\hline Age in years (mean \pm SD) & $47 \cdot 7 \pm 16 \cdot 6$ \\
Co-morbidity & \\
Lung dysfunction & $18(51 \%)$ \\
Heart dysfunction & $5(14 \%)$ \\
Malignancy & $3(8 \%)$ \\
Urogenital disorders & $1(3 \%)$ \\
Chronic renal failure & $2(6 \%)$ \\
Diabetes mellitus & $5(14 \%)$ \\
Haematological disorders & $1(3 \%)$ \\
Neurological disorders & $7(20 \%)$ \\
Abdominal disorders & $5(14 \%)$ \\
SAPS II on admission to ICU (mean \pm SD) & $40 \pm 3 \cdot 5$ \\
Prior hospitalization & $32(91 \%)$ \\
Catheters & \\
Urinary catheters & $35(100 \%)$ \\
Abdominal drainage & $1(3 \%)$ \\
Mechanical ventilation support & $33(94 \%)$ \\
Tracheostomy & $15(43 \%)$ \\
Gastrostomy & $3(8 \%)$ \\
Steroid treatment & $5(14 \%)$ \\
\end{tabular}

Twenty-nine of the $35(83 \%)$ patients were found to have colonization of the respiratory tract. Colonization of the respiratory tract occurred from day 6 to day 20 after admission to the ICU (median 13 days). The main colonizing pathogens (as defined in Methods) were Acinetobacter baumannii in 13 patients (38\% of the total number of studied patients), $P$. aeruginosa in ten patients $(28 \%)$, Enterobacter aerogenes in three patients (9\%), Klebsiella pneumoniae in two patients $(6 \%)$ and Stenotrophomonas maltophilia in one patient (3\%).

Forty-three imipenem-resistant Gram-negative bacteria were recovered from the 35 studied patients. Thirty-three of these bacteria were $P$. aeruginosa, five were $K$. pneumoniae and five were A. baumannii. The majority of the patients (60\%) who were colonized with $P$. aeruginosa harboured a strain resistant to carbapenems, aminoglycosides, monobactams, quinolones, ureidopenicillins and anti-pseudomonad cephalosporins (the strains were only sensitive to colistin).

A bla $a_{\mathrm{VIM}}$ gene was detected in nine out of $33(27 \%)$ imipenem-resistant $P$. aeruginosa isolates. All of these were isolated from six patients. Only one of the patients harbouring a $b l a_{\mathrm{VIM}}$ gene developed clinical infection. When specific primers for the whole $b l a_{\mathrm{VIM}-1}$ and $b l a_{\mathrm{VIM}-2}$ genes were used, only the latter gene was detected in all $b l a_{\mathrm{VIM}}$-positive isolates. The results of sequencing showed that the $865 \mathrm{bp} b \operatorname{la}_{\mathrm{VIM}}$ products were identical in all cases to the bla $_{\mathrm{VIM}-2}$ sequence available in the database (Gen Bank accession no. AF191564) (Walsh et al., 2005). No bla $a_{\mathrm{IMP}}$ gene was detected in any of the imipenem-resistant $P$. aeruginosa isolates. Testing for MBL genes showed negative results in all imipenem-resistant isolates of $K$. pneumoniae and A. baumannii.

In Table 2, we have presented the distribution of various possible risk factors in patients with or without colonization with $b a_{\mathrm{VIM}-2}$-carrying Gram-negative bacteria. Previous use of carbapenems $(P=0 \cdot 01)$ or other $\beta$-lactams (ceftazidime or piperacillin/tazobactam $)(P=0 \cdot 03)$ as well a stay in the ICU of $>20$ days $(P<0 \cdot 001)$ were associated with colonization with a $b l a_{\mathrm{VIM}-2}$-positive bacterium. Mortality was not different between patients in the two groups $(2 / 6$, $33 \cdot 3 \%$, versus $13 / 23,56 \cdot 5 \%$; $P=0 \cdot 39$ ).

\section{DISCUSSION}

Colonization of the respiratory tract of patients in this newly established ICU was common (83\%). Although our study lacked a control group of patients receiving care in a previously established ICU, comparison of our data with data from published historical controls suggests that there is no significant difference regarding colonization of the respiratory tract of patients who receive care in a newly established compared with a previously existing ICU (Jarvis et al., 1991). However, a point may be made about the relatively late occurrence of colonization of the respiratory tract of our patients (median 13 days) compared with the 
Table 2. Predictors of colonization with VIM-producing Gram-negative bacteria

Results are given as number of patients (\%), unless indicated otherwise.

\begin{tabular}{|c|c|c|c|}
\hline Characteristic & $\mathrm{VIM}^{+}(n=6)$ & $\operatorname{VIM}^{-}(n=23)$ & $P$ value \\
\hline Age in years $($ mean $\pm S D)$ & $49 \cdot 5 \pm 4 \cdot 1$ & $52 \cdot 1 \pm 3 \cdot 5$ & $0 \cdot 73$ \\
\hline No. of males & $4(67 \%)$ & $20(87 \%)$ & $0 \cdot 27$ \\
\hline \multicolumn{4}{|l|}{ Morbidity } \\
\hline Respiratory dysfunction & $3(50 \%)$ & $15(65 \%)$ & $0 \cdot 65$ \\
\hline Abdominal disorders & $2(33 \%)$ & $3(13 \%)$ & $0 \cdot 27$ \\
\hline Stroke & $1(17 \%)$ & $4(17 \%)$ & $0 \cdot 99$ \\
\hline Haematological disorders & $0(0 \%)$ & $1(4 \%)$ & $0 \cdot 99$ \\
\hline Immunosuppression & $1(17 \%)$ & $2(8 \%)$ & $0 \cdot 52$ \\
\hline SAPS II on admission to ICU (mean \pm SD) & $40 \pm 3 \cdot 5$ & $39 \pm 2 \cdot 6$ & $0 \cdot 18$ \\
\hline \multicolumn{4}{|l|}{ Treatment } \\
\hline Previous carbapenem administration & $5(83 \%)$ & $5(22 \%)$ & $0 \cdot 01$ \\
\hline Previous $\beta$-lactam administration & $6(100 \%)$ & $11(48 \%)$ & $0 \cdot 03$ \\
\hline Aminoglycosides & $5(83 \%)$ & $9(39 \%)$ & $0 \cdot 08$ \\
\hline Carbapenem/aminoglycosides & $5(83 \%)$ & $11(48 \%)$ & $0 \cdot 18$ \\
\hline Low albumin $\left(<3 \mathrm{~g} \mathrm{l}^{-1}\right)$ & $4(67 \%)$ & $12(52 \%)$ & $0 \cdot 66$ \\
\hline Gastrostomy & $2(33 \%)$ & $12(52 \%)$ & 0.65 \\
\hline Hospitalization $<20$ days & $0(0 \%)$ & $23(100 \%)$ & $<0 \cdot 001$ \\
\hline
\end{tabular}

observations of other investigators who studied patients in previously existing ICUs (median 6 days) (Kerver et al., 1988). It should be noted that other factors, such as patient load and duration of the ICU stay, as well as infection control practices, may influence these observations.

Bonten et al. (1994) demonstrated that colonization originates from exogenous sources, and not from the gastrointestinal tract as is often assumed. Trautmann et al. (2001) examined water faucets as a source of P. aeruginosa infection in a surgical ICU. All water outlets harboured distinct genotypes of $P$. aeruginosa over prolonged time periods in that study; of interest, over a period of 7 months, five out of 17 patients $(29 \%)$ were infected with $P$. aeruginosa genotypes that were also detectable in tap water. Johanson et al. (1972) reported that $22 \%$ of patients admitted to an ICU had their respiratory tract colonized with Gram-negative organisms over the first $24 \mathrm{~h}$. Kerver et al. (1988) showed that 40-60\% of mechanically ventilated patients were colonized, mainly in the lower respiratory tract, by ICU-acquired organisms after day 5 following ICU admission, with the percentage rising to $100 \%$ after day 10 .

Colonization with potentially pathogenic Gram-negative organisms from external sources occurs rapidly after hospital admission. The mechanism involved may be related to a loss of Gram-positive organisms attributable to the widespread use of antibiotics, as well as to hydrolysis of fibronectin, a common feature in severely ill patients. Loss of fibronectin facilitates the exposure of surface receptors on epithelial cells, thus allowing Gram-negative organisms to bind (Woods et al., 1981). However, there are other major contributing factors, such as impaired natural host-defence mechanisms as a result of underlying disease, and medical and surgical interventions.

MBLs are emerging worldwide as determinants of acquired antimicrobial resistance among nosocomial strains of Gram-negative bacteria. Our results suggest that $\beta$-lactams other than carbapenems may also select for MBL-producing strains in a single patient. It should also be noted that all imipenem-resistant Gram-negative strains were isolated from patients with at least 20 days of hospitalization in the ICU, indicating that a long stay in the ICU is associated with factors that lead to the acquisition of $b l a_{\mathrm{VIM}}$-positive bacteria. It is interesting that, in our study, colonization with MBL-producing Gram-negative bacteria did not seem to be associated with unfavourable patient outcome, although it should be emphasized that we studied a relatively small number of patients.

Our study is not without limitations. First, we did not directly compare the frequency and characteristics of colonization of patients in our newly established ICU with patients in another, previously existing ICU. In addition, our study focused only on Gram-negative bacteria, without examining colonization by Gram-positive bacteria and fungi. Also, we did not perform molecular clonality studies of the isolates and thus we cannot discern whether there was an outbreak situation during our study period.

In conclusion, despite the above limitations, our study provides information regarding colonization of the respiratory tract of patients in a newly established ICU by Gramnegative bacteria. In addition, our findings suggest that there is an association between exposure to carbapenems and other $\beta$-lactams, as well as a prolonged stay in the ICU, 
and the development of colonization by VIM-producing $P$. aeruginosa.

\section{ACKNOWLEDGEMENTS}

M. H. and S. L. designed the study and collected the data. N. J. L., M. K. and A. T. performed the microbiological studies. M. H. and M.E.F. did the statistical analysis and wrote the first draft of the manuscript. All authors made revisions of the manuscript and approved its final version.

\section{REFERENCES}

Andrade, S. S., Jones, R. N., Gales, A. C. \& Sader, H. S. (2003). Increasing prevalence of antimicrobial resistance among Pseudomonas aeruginosa isolates in Latin American medical centres: 5 year report of the SENTRY Antimicrobial Surveillance Program (1997-2001). J Antimicrob Chemother 52, 140-141.

Bonten, M. J. \& Weinstein, R. A. (1996). The role of colonization in the pathogenesis of nosocomial infections. Infect Control Hosp Epidemiol 17, 193-200.

Bonten, M. J., Gaillard, C. A., van Tiel, F. H., Smeets, H. G., van der Geest, S. \& Stobberingh, E. E. (1994). The stomach is not a source for colonization of the upper respiratory tract and pneumonia in ICU patients. Chest 105, 878-884.

de Champs, C., Poirel, L., Bonnet, R., Sirot, D., Chanal, C., Sirot, J. \& Nordmann, P. (2002). Prospective survey of $\beta$-lactamases produced by ceftazidime-resistant Pseudomonas aeruginosa isolated in a French hospital in 2000. Antimicrob Agents Chemother 46, 3031-3034.

Flaherty, J. P. \& Weinstein, R. A. (1996). Nosocomial infection caused by antibiotic-resistant organisms in the intensive care unit Infect Control Hosp Epidemiol 17, 236-248.

Itokazu, G. S., Quinn, J. P., Bell-Dixon, C., Kahan, F. M. \& Weinstein, R. A. (1996). Antimicrobial resistance rates among aerobic Gramnegative bacilli recovered from patients in intensive care units: evaluation of a national postmarketing surveillance program. Clin Infect Dis 23, 779-784.

Jarvis, W. R. (1996). The epidemiology of colonization. Infect Control Hosp Epidemiol 17, 47-52.

Jarvis, W. R., Edwards, J. R., Culver, D. H. \& 8 other authors (1991). Nosocomial infection rates in adult and pediatric intensive care units in the United States. National Nosocomial Infections Surveillance System. Am J Med 91 (Suppl. 2), S185-S191.

Johanson, W. G., Jr, Pierce, A. K., Sanford, J. P. \& Thomas, G. D. (1972). Nosocomial respiratory infections with Gram-negative bacilli. The significance of colonization of the respiratory tract. Ann Intern Med 77, 701-706.

Kerver, A. J., Rommes, J. H., Mevissen-Verhage, E. A., Hulstaert, P. F., Vos, A., Verhoef, J. \& Wittebol, P. (1988). Prevention of colonization and infection in critically ill patients: a prospective randomized study. Crit Care Med 16, 1087-1093.
Lauretti, L., Riccio, M. L., Mazzariol, A., Cornaglia, G., Amicosante, G., Fontana, R. \& Rossolini, G. M. (1999). Cloning and characterization of bla $_{\mathrm{VIM}}$, a new integron-borne metallo- $\beta$-lactamase gene from a Pseudomonas aeruginosa clinical isolate. Antimicrob Agents Chemother 43, 1584-1590.

Le Gall, J. R., Lemeshow, S. \& Saulnier, F. (1993). A new Simplified Acute Physiology Score (SAPS II) based on a European/North American multicenter study. JAMA ( $J$ Am Med Assoc) 270, 2957-2963.

Mavroidi, A., Tsakris, A., Tzelepi, E., Pournaras, S., Loukova, V. \& Tzouvelekis, L. S. (2000). Carbapenem-hydrolysing VIM-2 metallo$\beta$-lactamase in Pseudomonas aeruginosa from Greece. J Antimicrob Chemother 46, 1041-1042.

Poirel, L., Naas, T., Nicolas, D., Collet, L., Bellais, S., Cavallo, J.-D. \& Nordmann, P. (2000). Characterization of VIM-2, a carbapenemhydrolyzing metallo- $\beta$-lactamase and its plasmid- and integronborne gene from a Pseudomonas aeruginosa clinical isolate in France. Antimicrob Agents Chemother 44, 891-897.

Pournaras, S., Tsakris, A., Maniati, M., Tzouvelekis, L. S. \& Maniatis, A. N. (2002). Novel variant $\left(b / a_{\mathrm{VIM}-4}\right)$ of the metallo- $\beta$-lactamase gene bla $a_{\mathrm{VIM}-1}$ in a clinical strain of Pseudomonas aeruginosa. Antimicrob Agents Chemother 46, 4026-4028.

Pournaras, S., Maniati, M., Petinaki, E., Tzouvelekis, L. S., Tsakris, A., Legakis, N. \& Maniatis, A. N. (2003). Hospital outbreak of multiple clones of Pseudomonas aeruginosa carrying the unrelated metallo- $\beta$-lactamase gene variants $b l a_{\mathrm{VIM}-2}$ and $b l a_{\mathrm{VIM}-4}$. J Antimicrob Chemother 51, 1409-1414.

Senda, K., Arakawa, Y., Ichiyama, S., Nakashima, K., Ito, H., Ohsuka S. , Shimokata, K., Kato, N. \& Ohta, M. (1996). PCR detection of metallo- $\beta$-lactamase gene $\left(b l a_{\mathrm{IMP}}\right)$ in Gram-negative rods resistant to broad-spectrum $\beta$-lactams. J Clin Microbiol 34, 2909-2913.

Trautmann, M., Michalsky, T., Wiedeck, H., Radosavljevic, V. \& Ruhnke, M. (2001). Tap water colonization with Pseudomonas aeruginosa in a surgical intensive care unit (ICU) and relation to Pseudomonas infections of ICU patients. Infect Control Hosp Epidemiol 22, 49-52.

Tsakris, A., Pournaras, S., Woodford, N., Palepou, M.-F. I., Babini, G. S., Douboyas, J. \& Livermore, D. M. (2000). Outbreak of infections caused by Pseudomonas aeruginosa producing VIM-1 carbapenemase in Greece. J Clin Microbiol 38, 1290-1292.

Walsh, T. R., Toleman, M. A., Poirel, L. \& Nordmann, P. (2005). Metallo- $\beta$-lactamases: the quiet before the storm? Clin Microbiol Rev 18, 306-325.

Woods, D. E., Straus, D. C., Johanson, W. G., Jr \& Bass, J. A. (1981). Role of fibronectin in the prevention of adherence of Pseudomonas aeruginosa to buccal cells. J Infect Dis 143, 784-790.

Yan, J.-J., Hsueh, P.-R., Ko, W.-C., Luh, K.-T., Tsai, S.-H., Wu, H.-M. \& Wu, J. J. (2001). Metallo- $\beta \pm$-lactamases in clinical Pseudomonas isolates in Taiwan and identification of VIM-3, a novel variant of the VIM-2 enzyme. Antimicrob Agents Chemother 45, 2224-2228. 